\title{
Early endothelial damage and increased colonic vascular permeability in the development of experimental ulcerative colitis in rats and mice
}

\author{
Ganna Tolstanova ${ }^{1,2, *}$, Xiaoming Deng ${ }^{1,2}$, Samuel W French ${ }^{3}$, William Lungo ${ }^{3}$, Brankica Paunovic ${ }^{1}$, \\ Tetyana Khomenko ${ }^{1,2}$, Amrita Ahluwalia', Tessa Kaplan', Maria Dacosta-lyer ${ }^{1}$, Andrzej Tarnawski ${ }^{4,5}$, \\ Sandor Szabo ${ }^{1,2,6}$ and Zsuzsanna Sandor ${ }^{5,7}$
}

The role of endothelial damage and increased vascular permeability (VP) in the pathogenesis of ulcerative colitis (UC) has not been investigated. We examined using functional, morphologic, and molecular biologic studies whether and to what extent the endothelial barrier dysfunction precedes enhanced epithelial permeability (EP) and the development of mucosal lesions during the early stages of experimental UC. We showed that in rats with iodoacetamide (IA)-induced UC increased colonic VP occurs early (ie, 2.6 -fold increase at $15 \mathrm{~min}, P<0.01$ ) preceding changes in epithelial barrier permeability. EP was unchanged at 15 and $30 \mathrm{~min}$ after IA administration and was increased 1.9 -fold at $1 \mathrm{~h}$ and 6.7 -fold at $2 \mathrm{~h}$ (both $P<0.001$ ) after IA. In the dextran sodium sulfate-induced slowly developing UC, colonic VP was significantly increased in 2 days $(P<0.05)$ and EP only in 4 days $(P<0.05)$. Mucosal endothelial injury led to hypoxia $(P<0.05)$ of colonic surface epithelial cells $30 \mathrm{~min}$ after IA administration that was associated with increased expressions of transcription factors hypoxia-inducible factor- $1 \alpha$ and early growth response-1. Electron and light microscopy demonstrated areas of colonic mucosa with perivascular edema covered by intact layer of surface epithelial cells in both rat and mouse models of UC. This is the first demonstration in four models of UC that endothelial damage, increased colonic VP, perivascular edema, and epithelial hypoxia precede epithelial barrier dysfunction that is followed by erosions, ulceration, and inflammation in UC.

Laboratory Investigation (2012) 92, 9-21; doi:10.1038/labinvest.2011.122; published online 5 September 2011

KEYWORDS: endothelial damage; epithelial permeability; hypoxia; inflammatory bowel disease; ulcerative colitis; vascular permeability

Ulcerative colitis (UC) is characterized by chronic, recurrent ulcers and inflammation of the colonic mucosa. It has been postulated that impaired epithelial barrier function, resulting in penetration of bacteria and other antigens into the mucosa, is one of the major factors of UC pathogenesis. ${ }^{1-4}$

The epithelial barrier formed by a continuous layer of surface epithelial cells is important in preventing the penetration of bacteria and other antigens into the mucosa. The viability and integrity of the epithelial layer are critically dependent on oxygen and nutrients delivered by blood flow through the mucosal microvessels. Thus, vascular injury and diminished blood flow will result in tissue hypoxia and damage of epithelial cells. ${ }^{5}$

Anatomical and functional vascular changes in UC have been noted some time ago ${ }^{6,7}$ and confirmed recently. ${ }^{8}$ In early stages of UC, angiographic studies have demonstrated tortuous, dilated vessels, abnormal distribution, and small luminal irregularities in the peripheral branches. In contrast, advanced UC lesions demonstrated reduced vessel diameter, decreased vascular density, and diminished blood flow in the involved segments. ${ }^{6,7}$ Hatoum et $a l^{9}$ found in UC patients decreased vasodilatory capacity in chronically inflamed

\footnotetext{
'Diagnostic and Molecular Medicine, VA Long Beach Healthcare System, Long Beach, CA, USA; ${ }^{2}$ Department of Pathology, University of California-Irvine, Long Beach, CA, USA; ${ }^{3}$ Harbor-UCLA Medical Center, Los Angeles, CA, USA; ${ }^{4}$ Gastroenterology Health Care Groups, VA Long Beach Healthcare System, Long Beach, CA, USA;

${ }^{5}$ Department of Medicine, University of California-Irvine, Long Beach, CA, USA; ${ }^{6}$ Department of Pharmacology, University of California-Irvine, Long Beach, CA, USA and ${ }^{7}$ Internal Medicine, VA Long Beach Healthcare System, Long Beach, CA, USA

Correspondence: Dr S Szabo, MD, PhD, MPH, DSc (h.c.), Diagnostic and Molecular Medicine, Departments of Pathology and Pharmacology, VA Medical Center (05/113), VA Long Beach Healthcare System, 5901 East 7th Street, Long Beach, CA 90822, USA.

${ }^{*}$ Current address: Department of Pharmaco-Physiology, Kiev National Shevchenko University, Kiev, Ukraine.

Received 31 July 2010; revised 6 July 2011; accepted 8 July 2011
} 
mucosa, which was associated with high levels of oxidative stress. A study of trinitrobenzene sulfonic acid (TNBS)induced UC showed decreased colonic capillary blood flow in acute stage. ${ }^{10}$ Despite numerous reports on vascular alterations in UC, there are no parallel studies of the role of increased vascular permeability (VP) in the initiation and development of UC. The aim of this study was to determine whether the early endothelial injury and increased colonic VP with concomitant hypoxia are the primary events leading to changes in epithelial permeability (EP) and mucosal injury during the development of experimental UC.

Two chemically induced UC models characterized by a well-defined, time-dependent development of lesions were used to study sequential changes in vascular and EP preceding the development of mucosal erosions, ulcers, and infiltration with acute and chronic inflammatory cells. To determine the relevance of impaired endothelial barrier in UC, two models of spontaneously developing UC in interleukin (IL)-10 $10^{-/-}$ (ref. 11) and G-protein $\alpha$-inhibitor $2(\mathrm{G} \alpha-\mathrm{i} 2)^{-/-}$mice $^{12}$ were analyzed for early morphologic changes in colonic mucosa.

\section{MATERIALS AND METHODS \\ Animals}

Female Sprague-Dawley rats (170-200 g) were obtained from Harlan Sprague-Dawley (San Diego, CA, USA). IL-10 ${ }^{-1-}$ mice on a C57BL/6J background and wild-type C57BL/6J were obtained from Jackson Laboratory (Bar Harbor, ME, USA). $\mathrm{G} \alpha-\mathrm{i} 2^{-/-}$mice on a $129 \mathrm{~Sv} / \mathrm{C} 57$ background and wildtype 129Sv/C57 littermates of both sexes were provided by Dr Jonathan Braun's laboratory (UCLA, Department of Pathology). All animals were housed in the animal research facility at the VA Medical Center in Long Beach, CA, USA, under standard environmental conditions. All animals had unlimited access to Purina chow and tap water. These studies were approved by the Subcommittee for Animal Studies of the R\&D Committee of the VA Medical Center in Long Beach, CA, USA.

\section{lodoacetamide-Induced Colitis}

Experimental UC was induced in rats by the $\mathrm{SH}$ alkylator iodoacetamide (IA). ${ }^{13,14}$ This model is characterized by welldefined time-dependent changes in colon, for example, depletion of antioxidant glutathione, increased alkylation of protein $\mathrm{SH}$ followed by the development of erosions, ulcers, and infiltration of neutrophils as early as $6-12 \mathrm{~h}$ after administration of the chemical. ${ }^{13,14}$ This feature allowed us to detect very early changes, which precede the development of extensive colonic injury. Briefly, $0.1 \mathrm{ml}$ of $6 \%$ IA (Sigma, St Louis, MO, USA) dissolved in 1\% methylcellulose (Sigma) or the vehicle $1 \%$ methylcellulose was given to rats once by enema ( $7 \mathrm{~cm}$ from anus) via rubber catheter Nelaton S-8 (Rüsch, Germany). Rats were euthanized 5, 15, $30 \mathrm{~min}, 1,2$, 6 , and $12 \mathrm{~h}$ after intracolonic administration of IA. During autopsy, $7 \mathrm{~cm}$ of distal colon was removed for morphologic examination.

\section{Dextran Sodium Sulfate-Induced Colitis}

UC was induced in rats following the continuous administration of dextran sodium sulfate (DSS) (ICN Biochemicals, Solon, OH, USA) in drinking water. DSS, with a MW $36000-50000$ and a sulfur content of 15-20\%, was dissolved in water to provide a working dilution of $5 \%(\mathrm{w} / \mathrm{v})$ for oral consumption. In all, 5\% DSS solution was prepared fresh every day. Volume of ingested DSS solution was measured daily. Rats were euthanized 1, 2, 3, 4, and 5 days after $5 \%$ DSS consumption. At autopsy, $7 \mathrm{~cm}$ of distal colon was removed for morphologic examination.

\section{VP in IA- and DSS-Induced UC}

For the quantitative studies of colonic VP, Evans blue was used as described previously. ${ }^{15}$ Evans blue is a marker of albumin leakage since it binds tightly to albumin and it crosses endothelial barrier as the complex Evans blue/albu$\min (\mathrm{MW} \sim 67 \mathrm{kDa}) .{ }^{16}$ Rats were anesthetized with inhalation of isoflurane. Evans blue (1 mg/100 g in PBS; Sigma) was injected intravenously $15 \mathrm{~min}$ before autopsy that was performed 5, 15, $30 \mathrm{~min}$ and 1, 2, 6, and $12 \mathrm{~h}$ after $6 \%$ IA enema or vehicle (1\% methylcellulose). Rats with DSS-induced UC received intravenously Evans blue (1 mg/100 g in PBS) $15 \mathrm{~min}$ before autopsy that was performed on 1 (24 h), 2 (48 h), 3, 4, and 5 days after initiation of treatment with $5 \%$ DSS in drinking water. Evans blue was extracted from the colonic tissue $(7 \mathrm{~cm})$ using chloroform and measured by spectrophotometry at $610 \mathrm{~nm}$. Results were expressed as $\mathrm{mg}$ dye $/ \mathrm{g}$ wet weight of colon.

\section{EP in IA- and DSS-Induced UC}

To measure colonic EP, we used blood concentration of fluorescein isothiocyanate-conjugated (FITC)-dextran (MW $3.0-5.0 \mathrm{kDa}$; Sigma) after its intragastric administration. Before experiments, rats were fasted for $18 \mathrm{~h}$, but had unlimited access to water. Rats with IA-induced UC received by gavage $20 \mathrm{ml} / \mathrm{kg}$ of $\mathrm{PBS}(\mathrm{pH}=7.4)$ containing $22 \mathrm{mg} / \mathrm{ml}$ FITC-dextran $2 \mathrm{~h}$ before autopsy. Then the animals were euthanized at 15, $30 \mathrm{~min}$ and $1,2 \mathrm{~h}$ after $6 \%$ IA enema or vehicle ( $1 \%$ methylcellulose). Rats with DSS-induced UC were given by gavage $20 \mathrm{ml} / \mathrm{kg}$ of $\mathrm{PBS}(\mathrm{pH}=7.4)$ containing $22 \mathrm{mg} / \mathrm{ml}$ FITC-dextran $4 \mathrm{~h}$ before autopsy on days 1 ( $24 \mathrm{~h}$ ), 2 ( $48 \mathrm{~h}), 3,4$, or 5 after $5 \%$ DSS or vehicle (water) ingestion. Blood samples were obtained by cardiac puncture and were centrifuged ( 3000 r.p.m. at $\left.4^{\circ} \mathrm{C}\right)$ for $20 \mathrm{~min}$. Plasma $(100 \mu \mathrm{l})$ concentration of fluorescein was measured by using a microplate spectrofluorometer (NovoStar, BMG LABTECH, Durham, NC, USA) with excitation wavelength of $485 \mathrm{~nm}$ and an emission wavelength of $520 \mathrm{~nm}$ using serially diluted samples of the marker as standard.

\section{Tissue Hypoxia}

For detection of tissue hypoxia, we used Hypoxyprobe ${ }^{\mathrm{TM}}-1 \mathrm{Kit}$ (Natural Pharmacia International, Burlington, MA, USA). This method utilizes a small molecular marker, pimonidazole, 
which after intravenous injection forms adducts with thiol containing proteins only in oxygen-starved cells. Rats were anesthetized with inhalation of isoflurane. Pimonidazole-HCl $(6 \mathrm{mg} / 100 \mathrm{~g})$ was injected intravenously $90 \mathrm{~min}$ before autopsy; at 15, $30 \mathrm{~min}$ and $1,2 \mathrm{~h}$ after $6 \%$ IA or vehicle (1\% methylcellulose) enema. The removed distal colon $(7 \mathrm{~cm})$ was fixed in $10 \%$ buffered formalin and paraffinembedded sections ( $5 \mu \mathrm{m}$ thick) were immunostained using a mouse monoclonal antibody MAb1 (Natural Pharmacia International) that binds to protein adducts of pimonidazole in hypoxic cells. Sections were deparaffinized, hydrated, blocked for endogenous peroxidase using $3 \% \mathrm{H}_{2} \mathrm{O}_{2} / \mathrm{H}_{2} \mathrm{O}$, and subsequently subjected to microwave antigen retrieval using a Dako target retrieval solution (Dako, Carpinteria, CA, USA). An overnight incubation with mouse monoclonal antibody MAb1 at $4^{\circ} \mathrm{C}$ was followed by biotinylated secondary antibody and a peroxidase-labeled streptavidin-biotin, and then developed using the $\mathrm{ABC}$ detection method for examination under a Nikon microscope. To ensure specificity of the antibody, immunoabsorption of the antibodies was performed to provide appropriate control. The positive staining area was measured by MetaMorph 7.0 Videoimage Analysis System (Molecular Devices, Downington, PA, USA), and expressed as percentage of total mucosal area.

\section{Western Blotting}

At autopsy, the removed distal colon $(7 \mathrm{~cm})$ was cut along anti-mesenteric side and thoroughly rinsed in cold PBS. Colon was gently wiped by paper towel and flat by mucosa side up on ice. Using metal spatula, we gently scraped mucosa from the muscular layer. Total proteins $(100 \mu \mathrm{g})$, which were extracted from colonic mucosa in a lysis buffer containing protease inhibitors (Sigma), were processed routinely for western blotting as described previously. ${ }^{14}$ The primary antibodies were used against Egr-1 (1:500; Santa Cruz Biotech, Santa Cruz, CA, USA) and HIF- $1 \alpha$ (1:200, Novus Biologicals, LLC; Littleton, CO, USA). The loading controls were obtained by using antibody to $\beta$-actin (1:500; Santa Cruz Biotech). Every western blotting was repeated at least twice using proteins from three different rats/group. The density of western blotting bands was measured using the Eagle Eye II (Stratagene, Austin, TX, USA) and presented as relative density against density of $\beta$-actin bands.

\section{Electron and Light Microscopy}

Immediately after euthanasia, sections of colonic tissue were fixed in $2.5 \%$ glutaraldehyde in $0.1 \mathrm{M}$ PBS for $2 \mathrm{~h}$ at $22^{\circ} \mathrm{C}$. The specimens were then washed in $0.1 \mathrm{M}$ glycine, embedded in epoxy resin, and thick sections $(0.5-1 \mu \mathrm{m})$ were stained with toluidin blue for $30 \mathrm{~s}$ and washed in $0.1 \mathrm{M}$ PBS. Thin sections $(60-100 \mathrm{~nm})$ from selected area were stained with uranyl acetate/lead citrate for transmission electron microscopy. Electron micrographs were examined by two experienced pathologists who were unaware of the treatment (SWF, $\mathrm{WL}$ ). For light microscopy, 7-10 sections of distal colon from rats or mice were fixed in $10 \%$ buffered formalin, embedded in paraffin, cut at $5 \mu \mathrm{m}$, stained with hematoxylin and eosin, and coded for blind microscopic evaluation. Histologic slides were examined by two experienced pathologists who were unaware of the treatment (MD-I, SS).

\section{Statistical Analysis}

Quantitative results are expressed as mean \pm s.d. The statistical significance was determined by the non-parametric Mann-Whitney $U$-test, or Student's $t$-test where appropriate, and $P$-values of $<0.05$ were considered statistically significant.

\section{RESULTS}

Early Increase in Colonic VP in IA- and DSS-Induced UC Since IA-induced colitis is characterized by development of colonic erosions at $6-12 \mathrm{~h}$, our latest time point for this functional study was $12 \mathrm{~h}$ after IA enema. We found that Evans blue extravasations representing VP were visible at all time courses in colonic mucosa after IA administration (Figure 1a). Quantitative measurement of Evans blue extravasations in colonic mucosa demonstrated that colonic VP was significantly increased 2.6-fold $(P<0.01), \quad 2.3$-fold $(P<0.01), 3.2$-fold $(P<0.01)$, and 4.7 -fold $(P<0.001)$ at 15 , $30 \mathrm{~min}, 1$, and $2 \mathrm{~h}$ after IA enema, respectively, $v s$ control (Figure 1b).

In contrast to IA-induced colitis, DSS-induced colitis is characterized by the appearance of early clinical signs of colitis (eg, occult blood) at 3 days and the development of the first visible colonic lesions at 7 days after initiation of DSS treatment. ${ }^{17}$ Therefore, we measured colonic VP after 24, $48 \mathrm{~h}, 3,4$, and 5 days of DSS treatment. Since extravasated Evans blue can be easily visualized in the mucosa during autopsy, we noticed that in DSS-fed rats blue color was restricted to distal colon, but no other macroscopically apparent morphologic changes were present (data not shown). Measurements of extravasated Evans blue in colonic tissue demonstrated increased VP in three out of eight rats at $24 \mathrm{~h}$ after initiation of DSS treatment. The mean of this group was at the borderline of statistical significance $(P<0.06) v s$ control group. Significant increase in VP was noted on 2,3, and 4 days after initiation of DSS treatment $(P<0.05 v s$ control group) (Figure 1c).

\section{Increased Colonic EP in IA- and DSS-Induced UC}

In contrast to increased colonic VP, EP was not changed at 15 and $30 \mathrm{~min}$ after IA administration (Figure 1d). We detected significantly increased colonic EP (1.9-fold) only at $1 \mathrm{~h}$ $(P<0.001, v s$ control $)$ after IA enema, with further increase (6.7-fold) at $2 \mathrm{~h}(P<0.001$ vs control) (Figure 1d).

The severity and extent of DSS-induced colitis vary between rats, depending on volume of ingested water. Taking this into consideration we measured EP and VP in the same animals. We did not detect any EP changes in the first 3 days after initiation of DSS treatment, while VP was significantly 

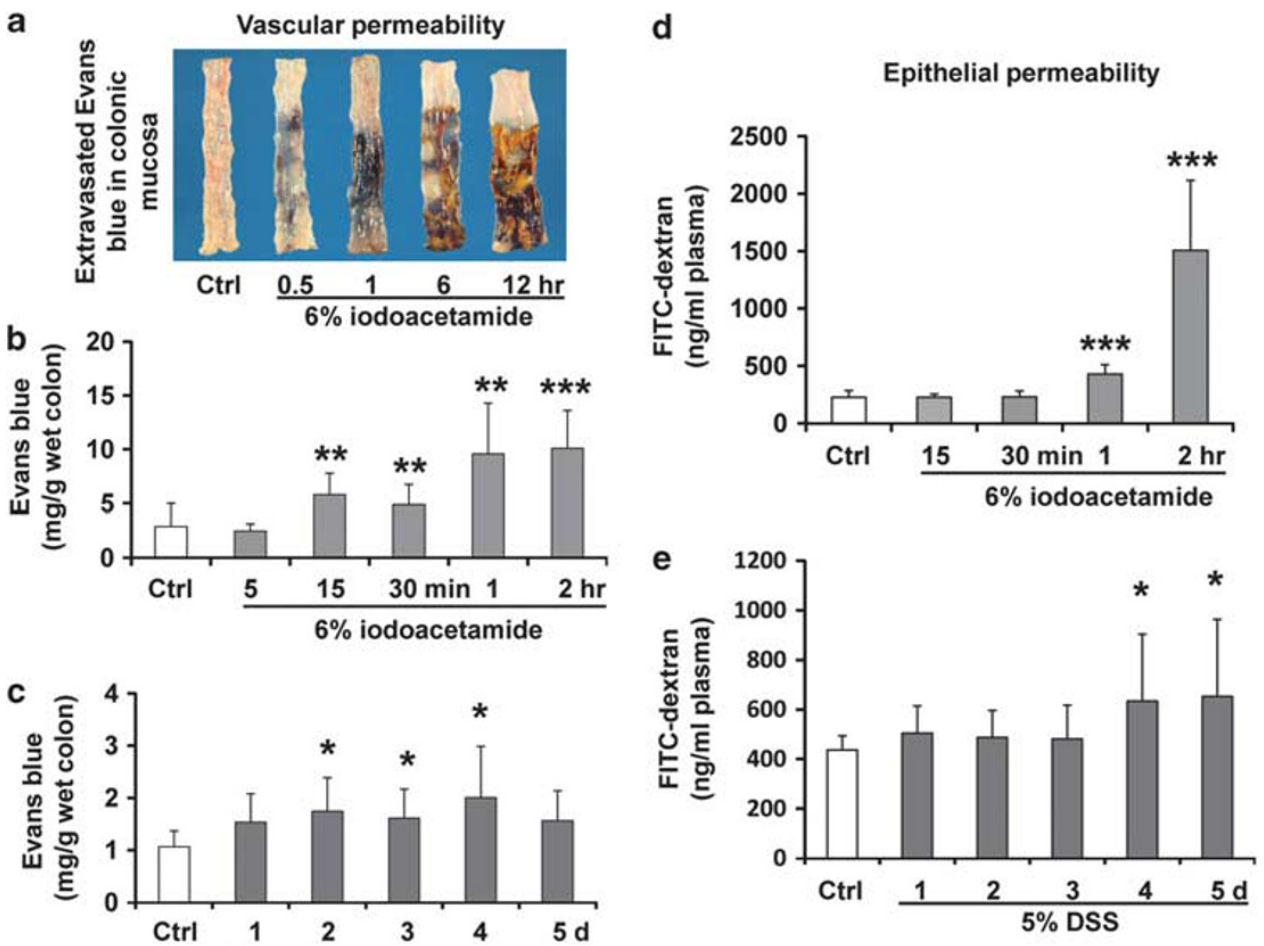

Figure 1 Increase in colonic VP precedes increased colonic EP during IA- or DSS-induced UC in rats. (a) Representative picture of Evans blue extravasations into colonic mucosa during IA-induced UC in rats. (b, c) Quantitative measurement of VP by extraction of extravasated Evans blue in the colonic mucosa. (d, e) Quantitative measurement of colonic EP by determining serum concentration of orally administered FITC-dextran. Results are expressed as mean \pm s.d.; $n=7$ rats/group. ${ }^{\star} P<0.05$; ${ }^{* \star P} P<0.01 ;{ }^{* *} P<0.001$ vs control (Ctrl).

increased at that time. Only 4 days after initiation of DSS treatment, we detected significantly increased colonic EP (1.4-fold, $P<0.05$ vs controls), with further increase 1.5 -fold after 5 days of DSS ingestion $(P<0.05)$ (Figure 1e).

\section{Hypoxia and Increased Expression of Hypoxia-Related Transcription Factors HIF-1 $\alpha$ and Egr-1 in IA-Induced UC} Since epithelial barrier is sensitive to hypoxia, we hypothesized that hypoxia in colonic mucosa during early stages of IA-induced UC precedes increased EP. To determine colonic mucosal hypoxia, we used a novel immunohistochemical method using a small molecular marker, pimonidazole (Hypoxyprobe- $1^{\mathrm{TM}}$ ), which allows visualizing hypoxia in tissue structures. ${ }^{18}$ As shown in Figure 2a, colonic samples from methylcellulose-treated group (control) showed some accumulation of pimonidazole protein adducts visualized immunohistochemically in superficial epithelial cells. This is consistent with oxygen gradient in the gut reported previously. ${ }^{19}$ Colonic samples from IA-treated groups revealed a marked hypoxia of surface epithelial cells as early as at $30 \mathrm{~min}$ after IA administration $(P<0.05$ vs control). Quantitative measurement of the positive staining areas demonstrated significantly increased hypoxic epithelial cells at $30 \mathrm{~min}$ and $1 \mathrm{~h}$ after IA (Figure $2 \mathrm{~b}$ ).

To further examine hypoxia in colonic mucosa of rats during the development of IA-induced UC, we determined protein expression of hypoxia-inducible transcription factors, for example, HIF-1 $\alpha$ and Egr-1. We found that significantly upregulated levels of HIF- $1 \alpha$ and Egr-1 (Figure 2c) were consistent with the increased hypoxia visualized by Hypoxyprobe-1 in colonic mucosa after IA administration.

\section{Electron Microscopy of Endothelial and Epithelial Changes in Colonic Mucosa in IA and DSS Models of UC} Transmission electron microscopic studies of rat colonic mucosa at $15,30 \mathrm{~min}$, and $1 \mathrm{~h}$ after IA administration demonstrated surface epithelial cells with intact microvilli (Figure 3). Subepithelial capillaries exhibited congestion (see red blood cells (white asterisks) in capillary lumens (black asterisks)), as early as $15 \mathrm{~min}$ (Figure $3 \mathrm{~b}$ ) after IA, associated with perivascular and inter-epithelial edema (arrows) (Figure 3b-d). High-magnification electron micrographs showed small breaks (arrowheads) in endothelial lining (Figure 4a, b, d, and f) and attached platelets, and platelets' aggregation (arrows) adhering to endothelial cells, indicating early endothelial cell injury (Figure $4 \mathrm{~d}$ and f) while at the same time the adjacent epithelial tight junctions (arrowheads) were intact (Figure 4e). Subsequently, platelets were also present in perivascular space (arrows), which was markedly enlarged and filled with fibrillar structures (most likely fibrin) (Figure 4c, d, and g). Intravascular congestion and edema were followed by separation of epithelial cells and 
a

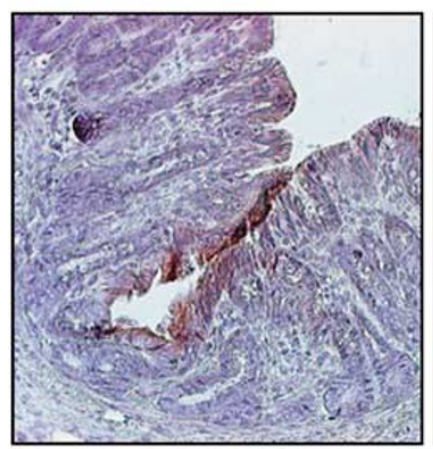

Control

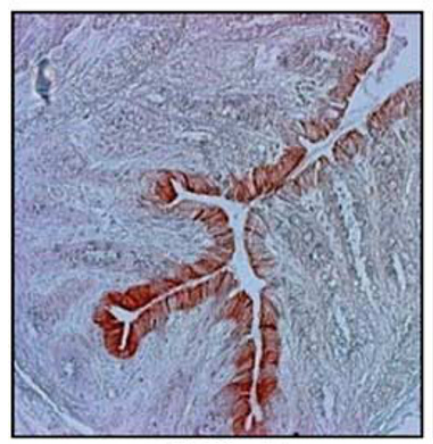

IA-30 min

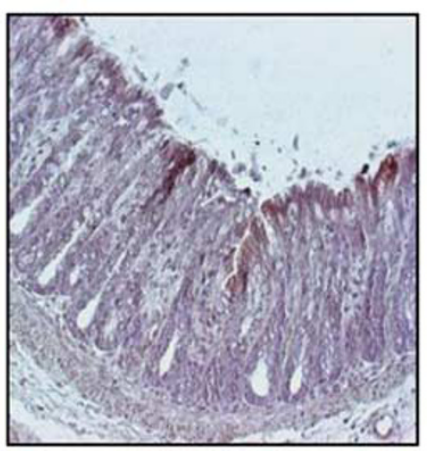

IA-15 min

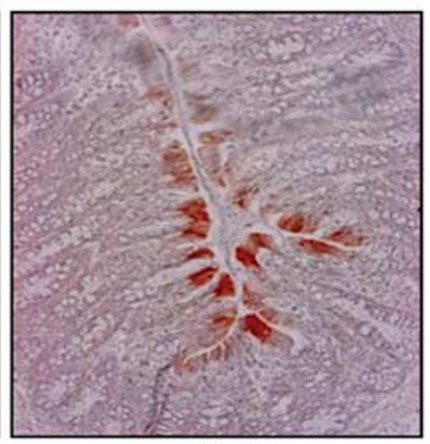

IA-1 hr

b

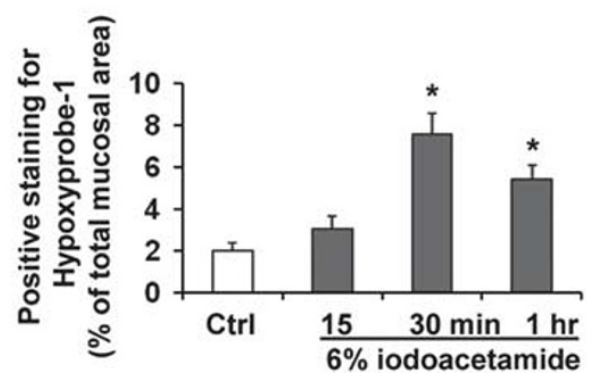

C
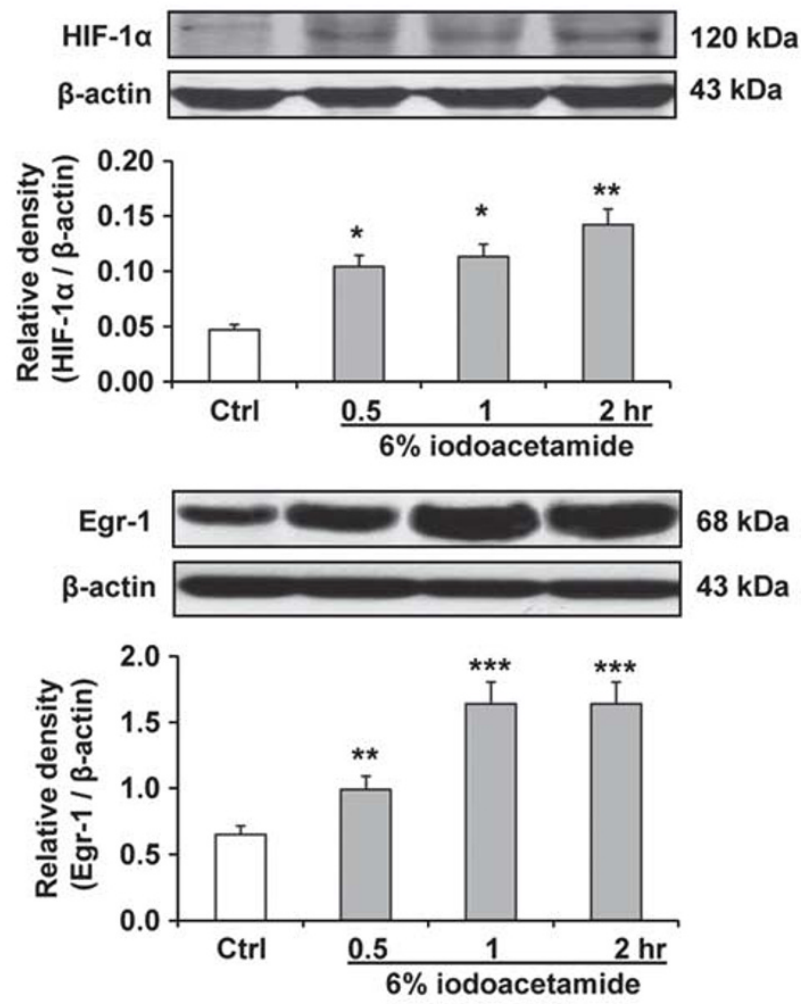

Figure 2 Colonic hypoxia and expression of hypoxia-related transcription factors HIF-1 $\alpha$ and Egr-1 in IA-induced UC. (a) Visualization of hypoxia in colonic mucosa by Hypoxyprobe-1 staining (brown staining, $\times 100$ ). IA administration markedly increased extent of staining in the surface epithelial cells, reflecting severe hypoxia in these cells. (b) Quantification of Hypoxyprobe- 1 staining. (c) Expressions of HIF-1 $\alpha$ and Egr-1 protein were detected by western blot. $\beta$-actin levels were used as loading controls. Assays were repeated two times with highly reproducible results. Results are expressed as mean \pm s.d. ${ }^{*} P<0.05 ;{ }^{* * P}<0.01 ;{ }^{* * * P}<0.001$ vs control (Ctrl).

extensive edema as illustrated in Figure 4. The edema most likely originated from subepithelial capillaries, since the inter-epithelial tight junctions were intact (arrowheads in Figures 3 and $4 \mathrm{e})$.

One hour after IA administration, at time when increased EP was detected (Figure 1d), an extensive perivascular edema containing fibrin deposits and intravascular platelet aggregations were present (Figures 3d and 4f). Most of the viable epithelial cells were separated by edema, while tight junctions (arrowheads) and intact microvilli remained intact (Figure 3d). In DSS model, electron microscopy also showed extensive perivascular inter-epithelial edema (arrows) at 24 and $48 \mathrm{~h}$ after DSS but surface epithelium and epithelial tight junctions were still intact (arrowheads) at 12,24, and $48 \mathrm{~h}$ after initiation of DSS treatment (Figure 5b-d). As an indication of endothelial damage, platelets were attached to endothelium (arrows, Figure 5E1 and $\mathrm{f}$ ), but often extravasated platelets were also seen (arrows, Figure 5E2). After $48 \mathrm{~h}$ of DSS ingestion (Figure $5 \mathrm{~g}$ and $\mathrm{h}$ ), subepithelial capillary endothelial cells showed swelling and disintegration (circled and squared areas), surrounded by perivascular edema (black asterisks), often containing extensive fibrin deposit (arrowheads).

\section{Light Microscopy of Colonic Mucosa in IA- and DSS-Induced UC}

Light microscopic study on colonic tissue thick sections (which were used for transmission electron microscopy) showed gradually increased edema in subepithelial areas (arrows) at 15, $30 \mathrm{~min}$, and $1 \mathrm{~h}$ after IA enema (Figure 6b-d, 
a

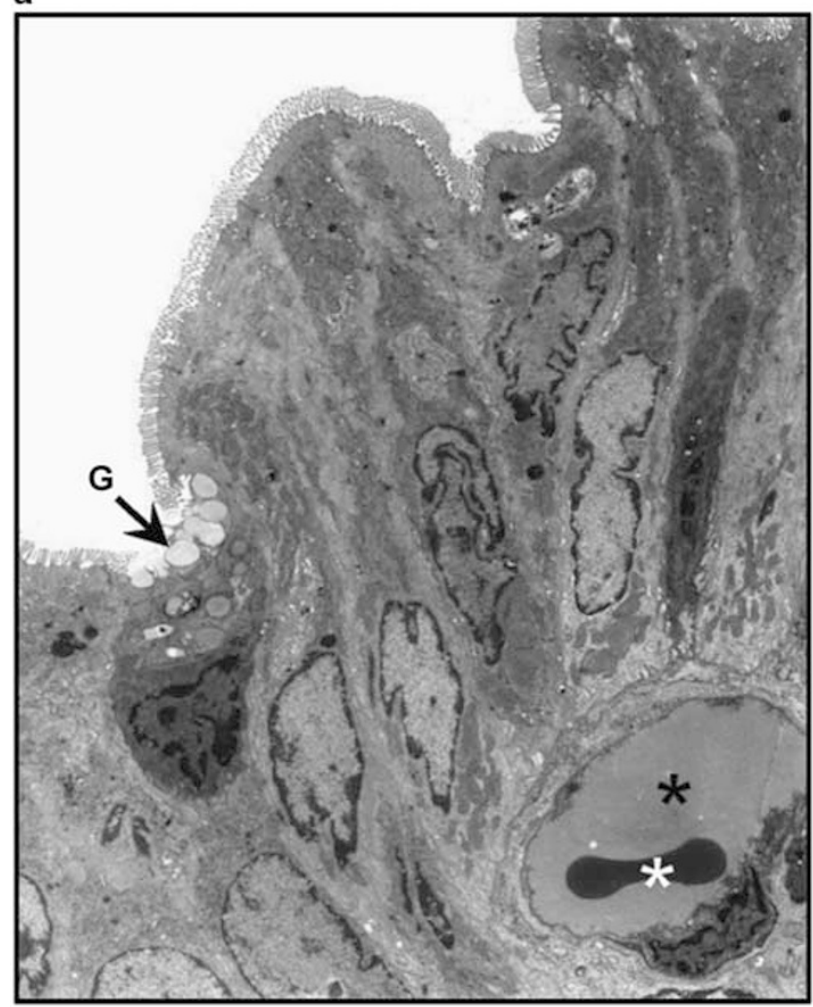

Ctrl $(5000 \mathrm{X})$

\section{C}

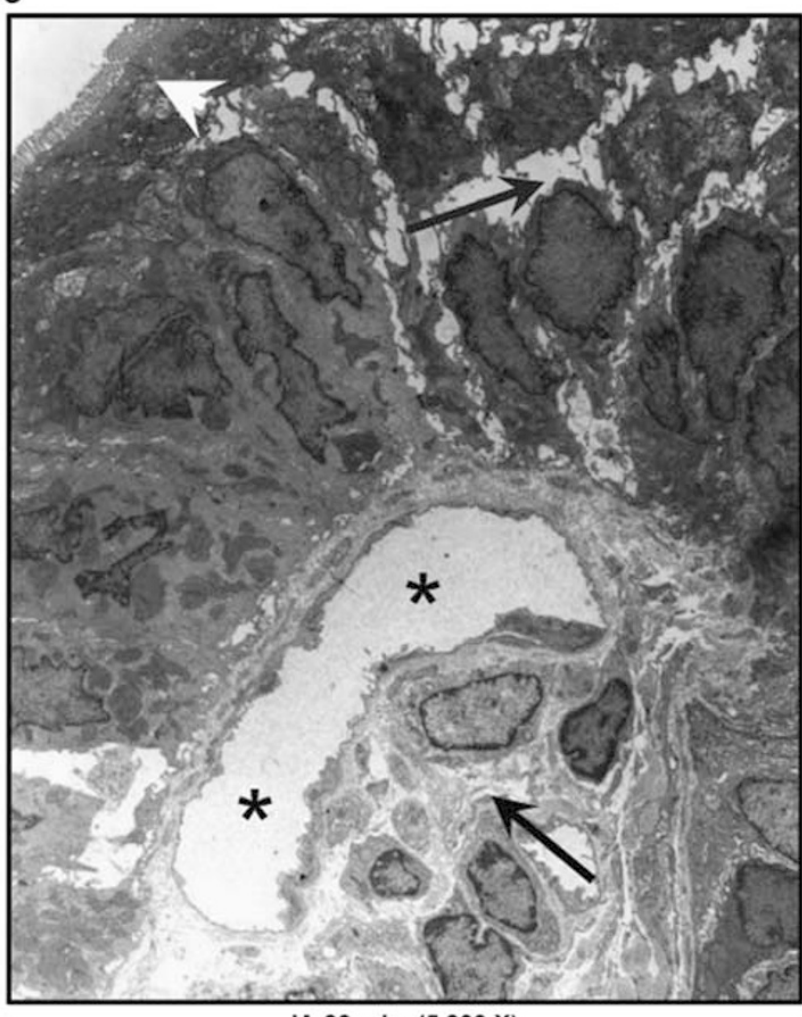

IA-30 min $(5000 X)$ b

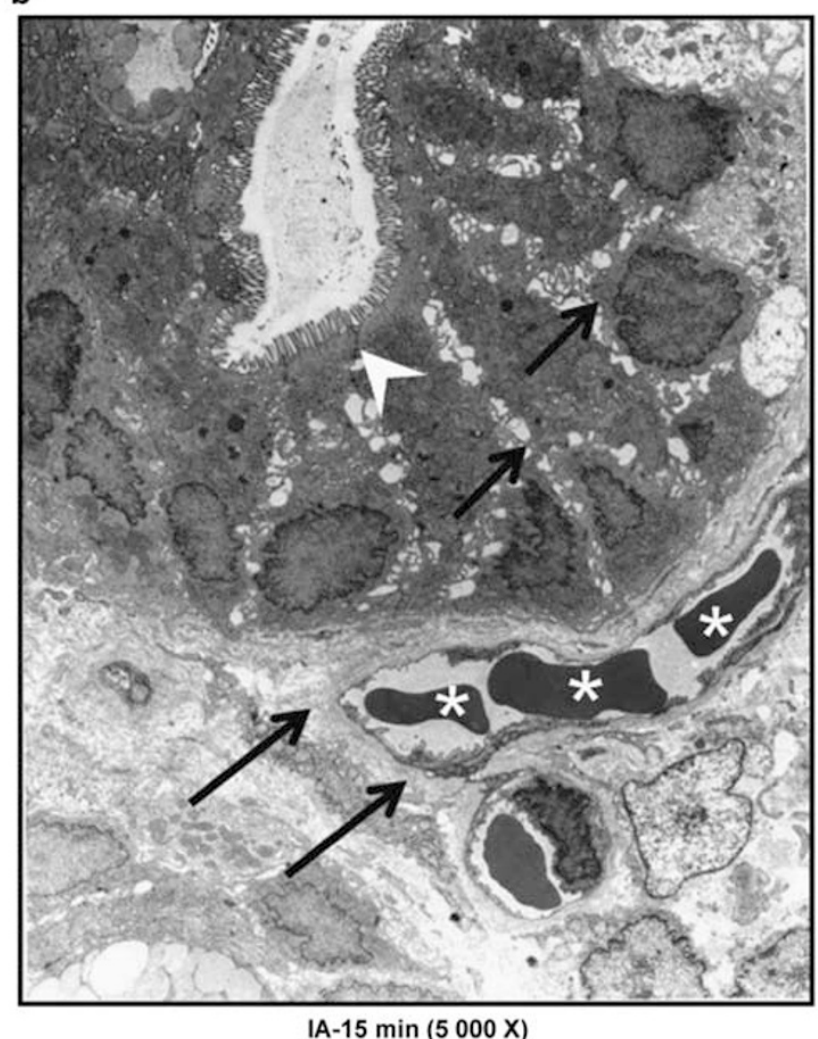

d

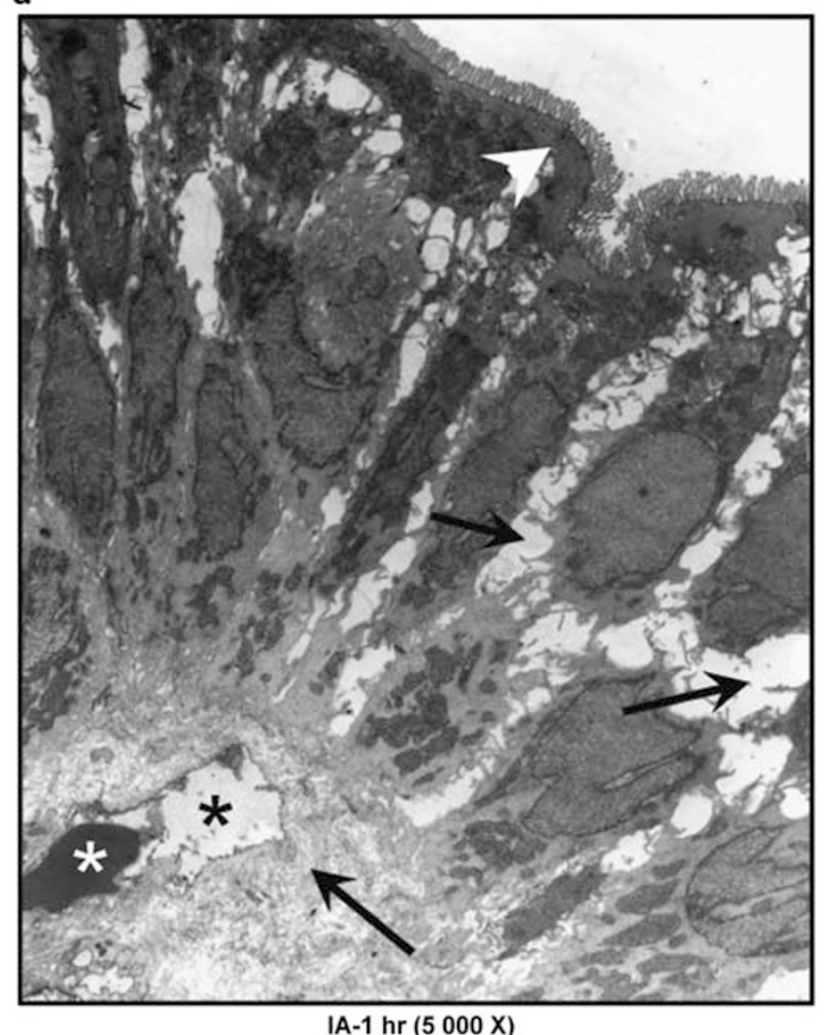


respectively), but the surface epithelial cell layer remained intact (arrowheads) with no inflammatory cells. Light microscopy also indicated that a focal, mild lamina propria edema (arrow) was present at $24 \mathrm{~h}$ (Figure $7 \mathrm{~b}$ ) followed by an extensive submucosal edema (double-headed arrow) at $48 \mathrm{~h}$ (Figure 7c) after initiation of DSS and a focal lamina propria inflammation in 3 days (Figure $7 \mathrm{~d}$ ) when the surface epithelial cell layer was still intact (Figure 7b-d). Similar changes were seen in 4 days (Figure 7e) after DSS ingestion. Only in 5 days after initiation of DSS treatment, colonic mucosal erosions were present (Figure 7f).

\section{Edema in Colonic Mucosa in IL-10 ${ }^{-1-}$ and $\mathbf{G} \alpha-\mathrm{i2}^{-1-}$ Mice Spontaneously Developing UC}

To determine the relevance of our findings in chemically induced models of UC, we analyzed histologic changes in colonic mucosa of $\mathrm{G} \alpha-\mathrm{i} 2^{-1-}$ and IL- $10^{-1-}$ mice of different ages with spontaneously developing UC.

We demonstrated that the earliest histologic changes in these spontaneously developing UC mice were subepithelial edema, later followed by edema involving a part or the entire lamina propria (Figure 8, arrows), often containing chronic inflammatory cells (Table 1). Areas of colonic mucosa with

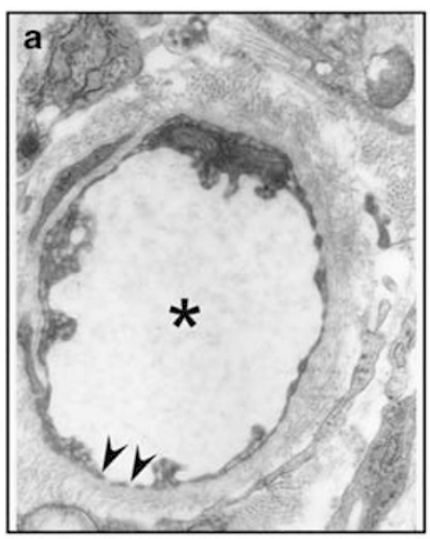

IA-15 $\min (30000 X)$



IA-15 $\min (12500 \mathrm{X})$

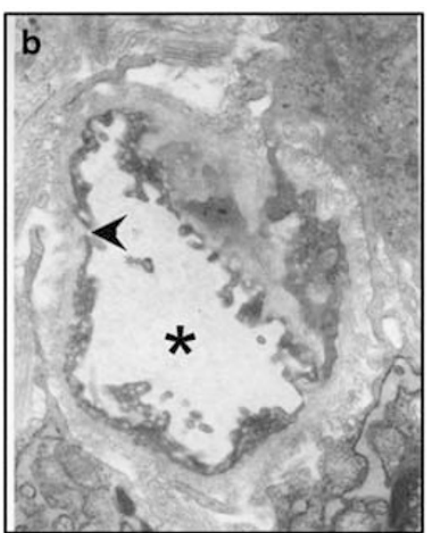

IA-15 $\min (25000 X)$

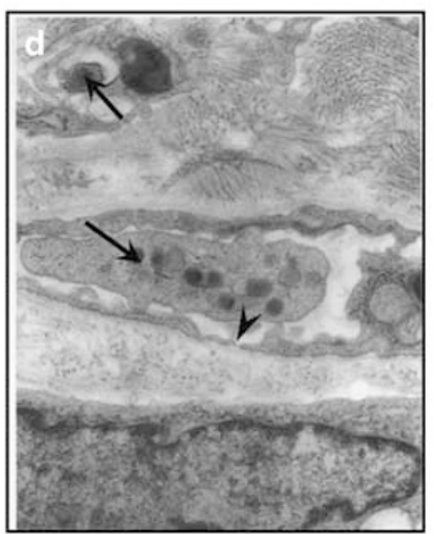

IA-15 $\min (30000 \mathrm{X})$

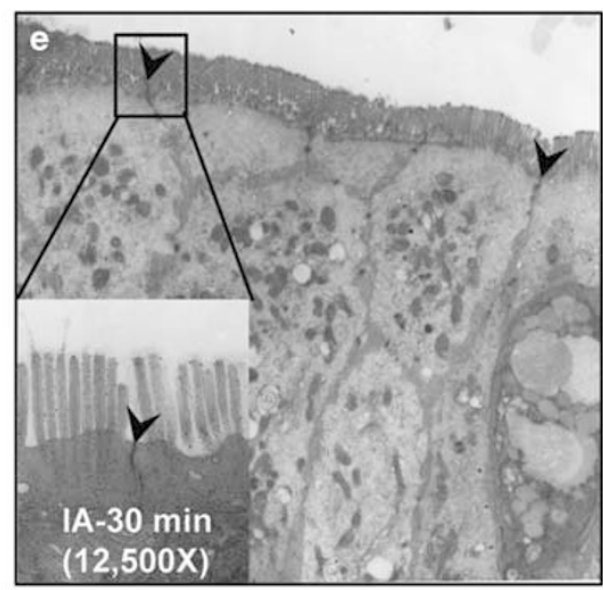

IA-30 $\min (7500 \mathrm{X})$

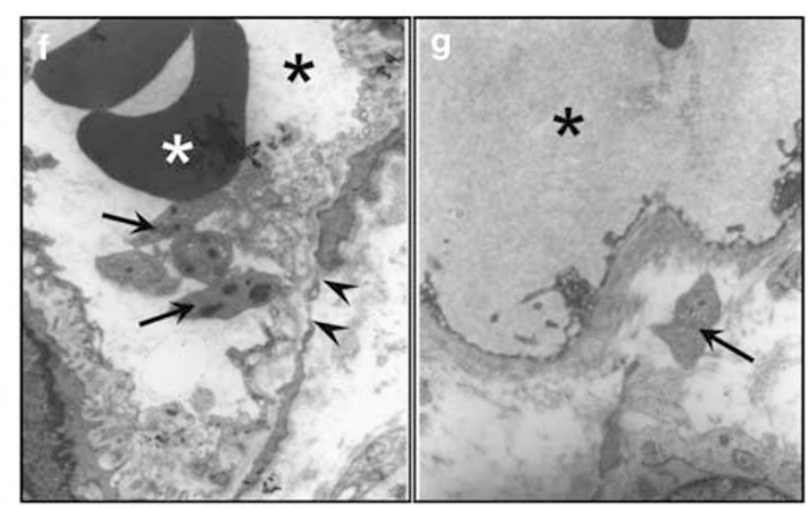

IA-1 hr (15 000 X)
IA-1 hr (25000 X)

Figure 4 High-magnification TEM of colonic mucosa during the development of IA-induced UC. (a-d) The electron micrographs show small breaks in endothelial lining (arrowheads) and attached platelets adhering to endothelial cells (arrows), with some extravascular platelets (arrows). The perivascular spaces are markedly enlarged and filled with fibrillary proteins, most likely originating from capillaries. (e) There are normal surface epithelial cells of colonic mucosa with intact tight junctions (arrowheads). (f, g) After IA, the TEM shows breaks in endothelial lining (arrowheads), an extensive perivascular edema containing fibrin deposits, intravascular platelet aggregation (arrow in f) and extravascular platelets (arrow in g). Capillary (black asterisks), red blood cells (white asterisks).

Figure 3 Representative transmission electron micrographs (TEMs) of colonic mucosa during development of IA-induced UC. Electron microscopy (uranyl acetate/lead citrate staining, $\times 5000)$. (a) TEM of control colon revealed densely packed surface epithelial cells, with clearly identified microvilli. On the bottom of section, there is a capillary (black asterisks) lined with endothelial cells. Capillary lumen contains red blood cells (white asterisks). Perivascular spaces are tightly packed and no intra- and extravascular platelets can be seen. There are goblet cells (G) - the top left of the picture. (b-d) Colonic mucosa of rats at 15, $30 \mathrm{~min}$, and $1 \mathrm{~h}$ after IA enema reveals some separation of epithelial cells but intact microvilli and tight junctions (arrowheads). Prominent perivascular edema with extravasated platelets is present at $30 \mathrm{~min}$. Extensive perivascular edema containing fibrin deposits (arrows). 


\section{Colonic epithelial barrier}

a

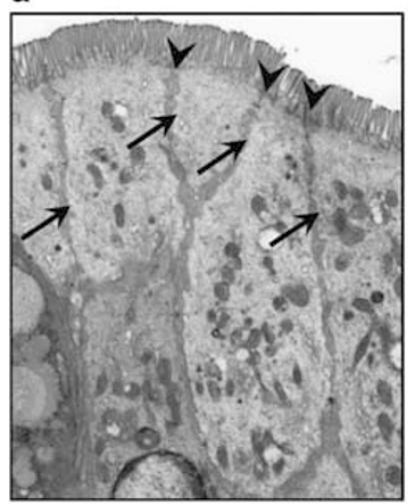

Control $(7500 \mathrm{X})$

c

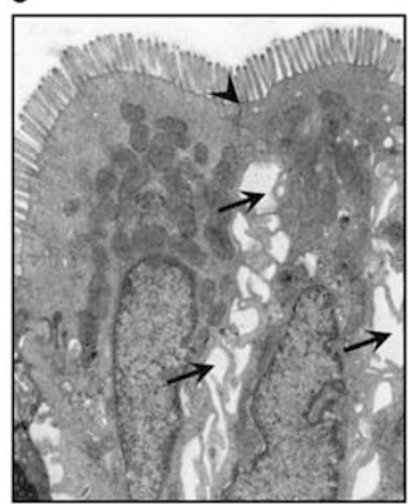

DSS $24 \mathrm{hr}(7500 \mathrm{X})$ b

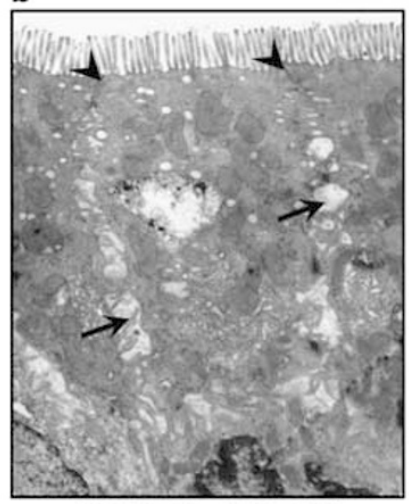

DSS $12 \mathrm{hr}(7500 \mathrm{X})$

d



DSS $48 \mathrm{hr}(7500 \mathrm{X})$

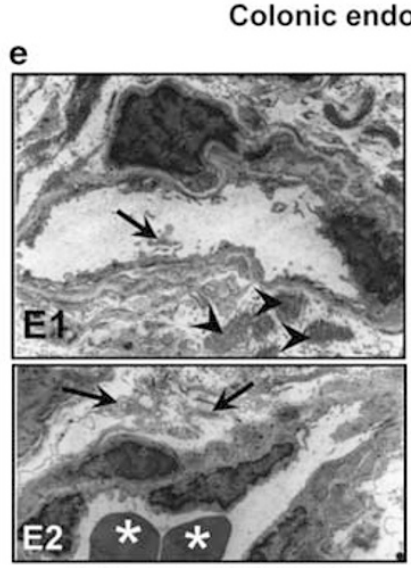

DSS $12 \mathrm{hr}(10000 \mathrm{X})$

g



DSS $48 \mathrm{hr}(8750 \mathrm{X})$

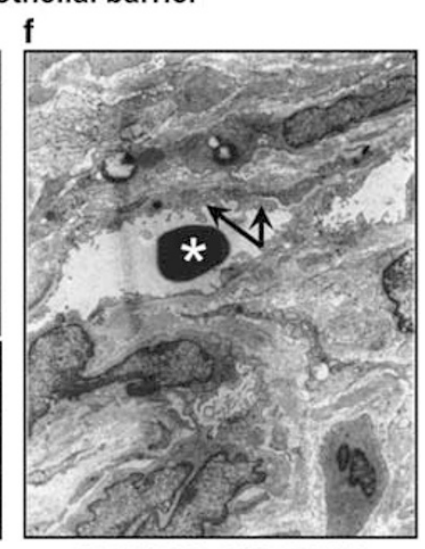

DSS $24 \mathrm{hr}(10000 \mathrm{X})$

h



DSS $48 \mathrm{hr}(25000 \mathrm{X})$

Figure 5 TEM of colonic tissue during development of DSS-induced UC. (a-d) Colonic epithelial barrier. (a) Intact inter-epithelial tight junctions (arrowheads) and adherens junctions in the colonic mucosa of control animals. (b-d) There is edema between epithelial cells (arrows) but the microvilli and epithelial tight junctions are still intact (arrowheads) at 12, 24, and $48 \mathrm{~h}$ of DSS ingestion. (e-h) Colonic endothelial barrier.

(e, f) Attached platelets to endothelium (arrows in E1, f) and extravasated platelets (arrows in E2). (g, h) Swelling and disintegration (circled and squared areas) of subepithelial capillary endothelial cells surrounded by perivascular edema (black asterisks), containing extensive fibrin deposit (arrowheads). White asterisks: red blood cells.

perivascular edema covered by intact layer of surface epithelial cells were present (arrowheads) in both $\mathrm{G} \alpha-\mathrm{i} 2^{-1-}$ and IL-10 $0^{-1-}$ mice (Figure 8 ). These changes were seen in relatively young mice that did not have signs of colitis and in advanced stages of UC in older mice as well. These changes were adjacent to well-developed erosions or ulcers, but were also present in otherwise uninvolved areas of colon. Relatively few erosions and ulcers were present in both genetic models of UC in mice (Table 1).

\section{DISCUSSION}

The present study demonstrated for the first time that endothelial damage, impaired endothelial barrier function, and increased colonic VP precede the changes in colonic epithelial barrier function and the development of colonic lesions in all the four investigated models of experimental UC. We also demonstrated that hypoxia of colonic surface epithelial cells precedes increased EP in IA-induced UC model. Consistent with hypoxic changes, the expression of hypoxia-related transcription factors HIF- $1 \alpha$ and Egr-1 were significantly upregulated in the colonic mucosa in IA-induced UC.

UC is a chronic inflammatory disorder, which is characterized by repeated cycles of colonic mucosal damage/ ulceration and regeneration. Although the pathogenesis of UC is not fully understood, the impairment of the epithelial barrier function has been recently shown to play important pathophysiologic role. ${ }^{2}$ As a consequence of impaired epithelial barrier integrity, enteric bacteria and other antigens may cross the epithelial lining, penetrate into mucosa leading to an enhanced immune response, and initiate and/or perpetuate inflammatory reaction in immunologically or genetically predisposed patients. ${ }^{20}$

Intestinal permeability is influenced not only by the epithelial but also by the microvascular endothelial barriers. ${ }^{21}$ Our laboratory was the first to demonstrate that microvascular 


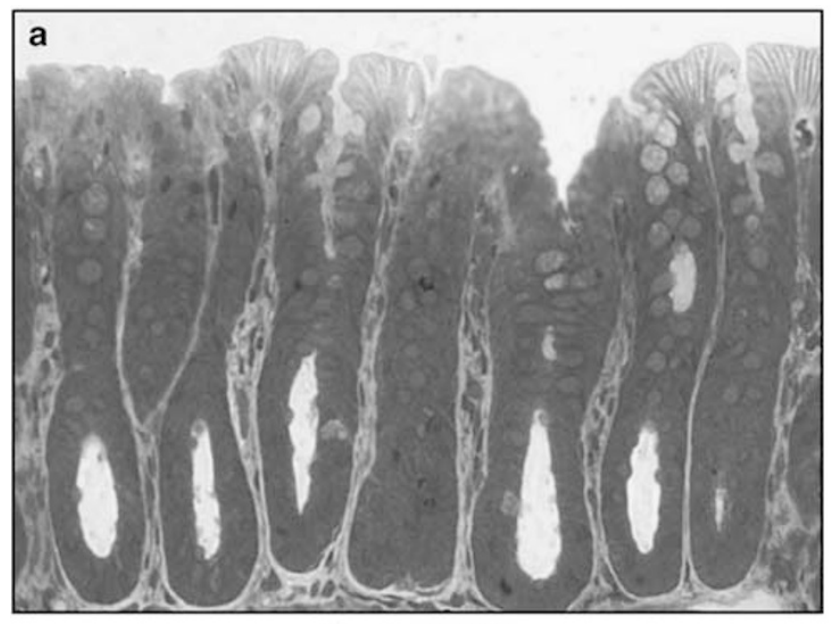

Control (200 X)

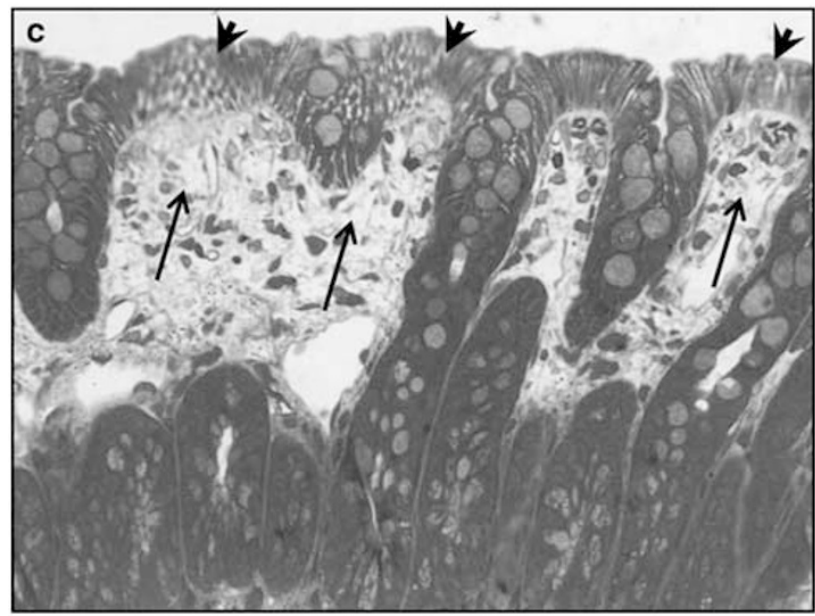

IA-30 min (200X)



IA-15 $\min (200 X)$



IA-1 hr (200 X)

Figure 6 Representative photomicrographs of colonic mucosa during development of IA-induced UC. Light microscopy (toluidin blue staining, $\times 200$ of sections, $0.5-1.0 \mu \mathrm{m}$ ) was used for electron microscopy study. (a) Control. There is a gradual increase of subepithelial edema at 15 (b), $30 \mathrm{~min}(\mathbf{c})$, and $1 \mathrm{~h}$ (d) after IA enema, while surface epithelial cells layer remained intact and no acute inflammatory cells were seen.

injury, leading to increased VP and capillary stasis, precedes the development of mucosal erosions and ulcers in the stomach and duodenum. ${ }^{22,23}$ Recent publications on the role of intestinal permeability mostly focus on disrupted epithelial barrier integrity in the pathogenesis of UC. ${ }^{1-4,24}$ In contrast, the role of microvascular endothelial barrier in defense of intestinal homeostasis is less recognized and investigated despite the fact that it: (1) is essential for capillary blood flow, oxygen and nutrient delivery; (2) serves as a 'gatekeeper' preventing the extravasation of circulating leukocytes and other inflammatory cells and proteins into the mucosa; and (3) has a major role in innate immune response, by synthesis and release of pro-inflammatory cytokines. ${ }^{10,25-27}$

We found that young $\mathrm{G} \alpha-\mathrm{i} 2^{-/-}$mice in early stages of spontaneously developing UC demonstrate areas of colonic mucosa with perivascular edema in the lamina propria covered by uninterrupted normal layer of surface epithelial cells. Older $\mathrm{G} \alpha-\mathrm{i} 2^{-/-}$mice with advanced stage of UC had focal areas of perivascular edema containing leukocytes and a continuous layer of surface epithelial cells outside the areas of erosions and ulcers. Despite the fact that we found a significant difference in histologic injury score of colonic mucosa between 10 - and 12 -week-old IL-10 ${ }^{-1-}$ mice, the areas of colonic mucosa with perivascular edema in the lamina propria were fully covered by normal layer of surface epithelial cells in all age groups. Based on these observations, we hypothesized that endothelial injury and increased VP precede the epithelial barrier disruption and might be a critical early step in disease pathogenesis. Our findings are in agreement with recent studies in T-cell receptor $\alpha$ genedeficient mice developing intestinal inflammation despite non-disrupted intestinal epithelial barrier. ${ }^{28}$ In these mice, an increase in intestinal IFN- $\gamma$ levels, which may increase VP, ${ }^{29}$ 


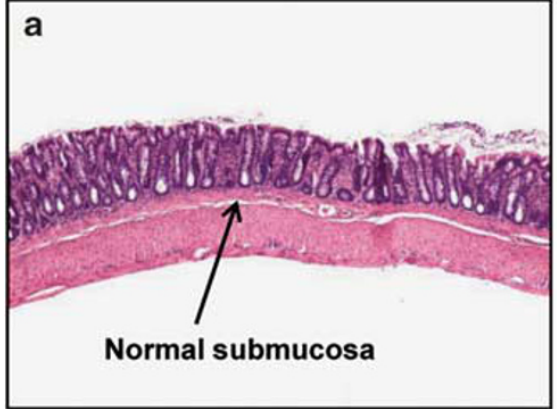

Control

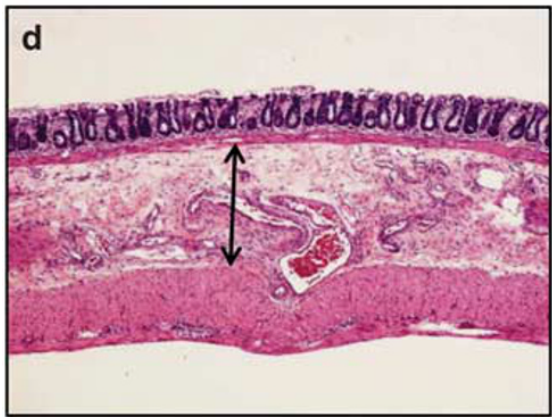

DSS-3 days

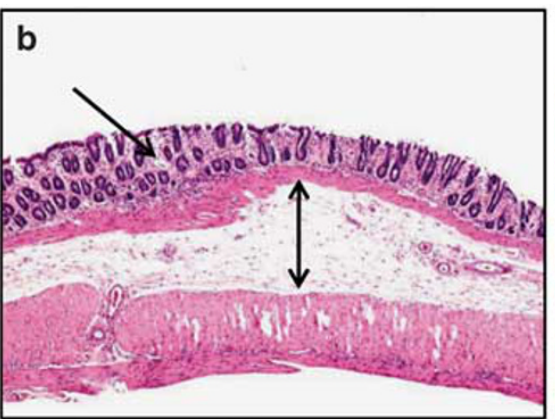

DSS-1 day

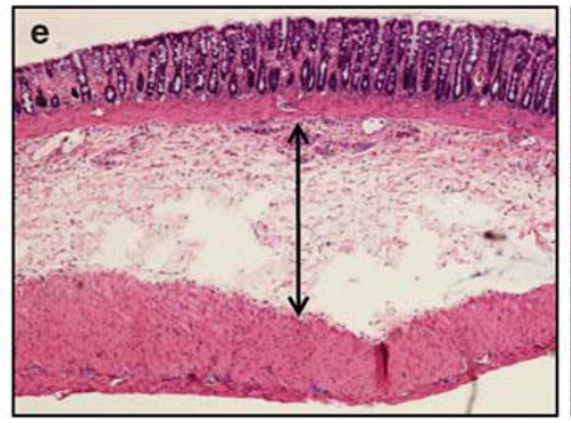

DSS-4 days

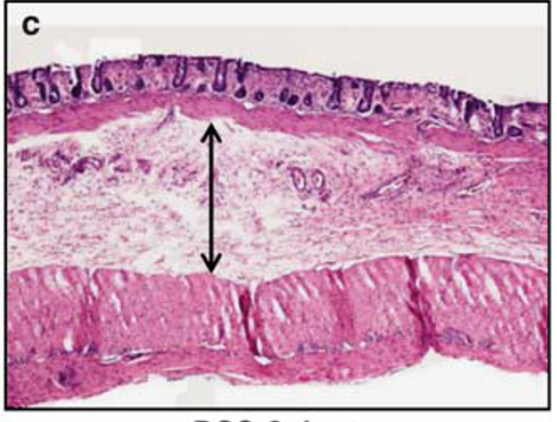

DSS-2 days

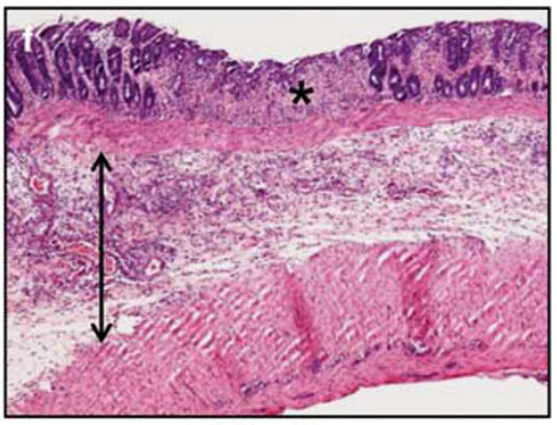

DSS-5 days

Figure 7 Light microscopy of colonic mucosa in DSS-induced UC in rat (H\&E staining, $\times 40$ ). (a) Control. Focal lamina propria edema present at $24 \mathrm{~h}$ (b), an extensive submucosal edema at $48 \mathrm{~h}$ (c), and submucosal edema in 3 days (d) after initiation of DSS treatment. The surface epithelial cells layer remains intact. Similar changes are seen after DSS ingestion for 4 days (e). Only after 5 days of DSS ingestion mucosal necrosis (erosion) and extensive submucosal inflammation are present (f).

preceded the onset of mucosal inflammation. Also, transgenic mice, which had significantly increased EP because of constitutively expressed active myosin light chain kinase specifically within intestinal epithelial cells, did not develop spontaneous UC, despite mucosal immune activation, ${ }^{30}$ possibly, because of intact endothelial barrier.

In our mechanistic study using chemically induced experimental UC, in which sequence of events can be studied, since the lesions develop in a predictable manner, we found an early increase of colonic VP and endothelial cell injury before changes in EP and development of colonic lesions. Transmission electron microscopic studies confirmed that microvascular changes were present before EP was increased and that the epithelial tight junctions remained intact despite the underlying lamina propria edema of vascular origin. Mori et $a l^{31}$ found a similar rapid onset of endothelial cell dysfunction (on day 2), manifested by increased VP, in mice with $3 \%$ DSS-induced UC that was accompanied by adherent platelets and leukocytes.

One of the consequences of vascular damage is the development of hypoxia, which in turn may induce intestinal epithelial cell injury by increased level of oxygen-derived toxic free radicals and production of inflammatory mediators. ${ }^{32,33}$ Wang et $a l^{34}$ showed that exposure of Caco-2 cell monolayer to hypoxia significantly lowered transepithelial electrical resistance, decreased protein expressions of
ZO-1, phosphorylated myosin light chain and myosin light chain kinase, thus imparing epithelial barrier. Moreover, Synnestvedt et $a l^{35}$ demonstrated that HIF- $1 \alpha$ is active in intestinal epithelia, associated with increased intestinal permeability in murine hypoxia model and decreased transepithelial electrical resistance of T84 epithelial cells under hypoxia with concomitant activation of HIF-1-dependent regulatory pathways. A number of clinical and experimental studies showed oxygen deprivation of colonic epithelial cells in inflammatory bowel disease (IBD).$^{5,10,25,36}$ Moreover, prevention of hypoxia by heparin ${ }^{37}$ or endothelin-1 receptor antagonist $^{38}$ significantly reduced the degree of mucosal injury and inflammatory response. In addition to endothelial damage and intravascular congestion, our present study demonstrated hypoxia in colonic surface epithelial cells that preceded the enhanced colonic EP. Recent studies of Colgan and Taylor showed a significant retention of nitroimidazole (hypoxia marker) in both superficial and deeper regions of colonic mucosa in mice with TNBSinduced UC at 7 days compared with the mucosal nitroimidazole retention only in the surface epithelial cells in the vehicle-treated control mice. ${ }^{5,39}$ Our study also showed Hypoxyprobe-positive staining in the surface epithelial cells in vehicle-treated control rats. However, unlike Colgan and Taylor's study, our results did not show deeper mucosal hypoxia in UC rats, because we examined the hypoxia of 


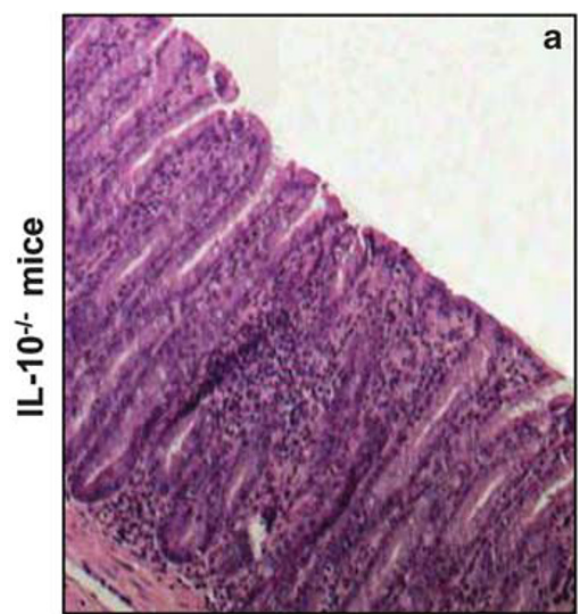

WT control

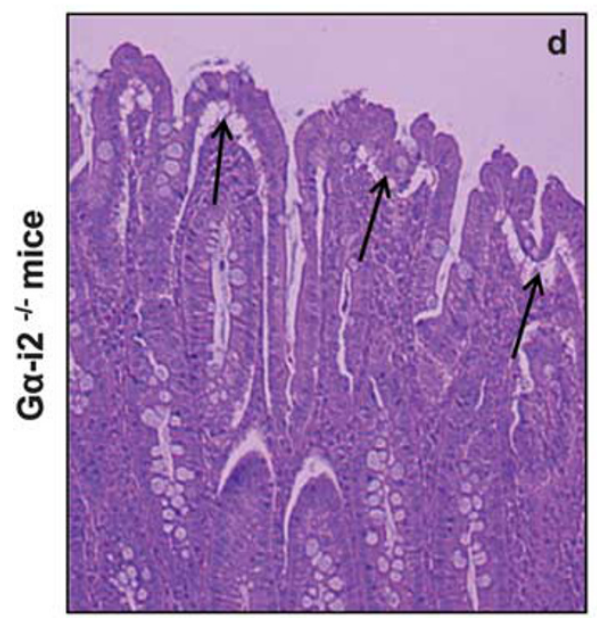

6 weeks old

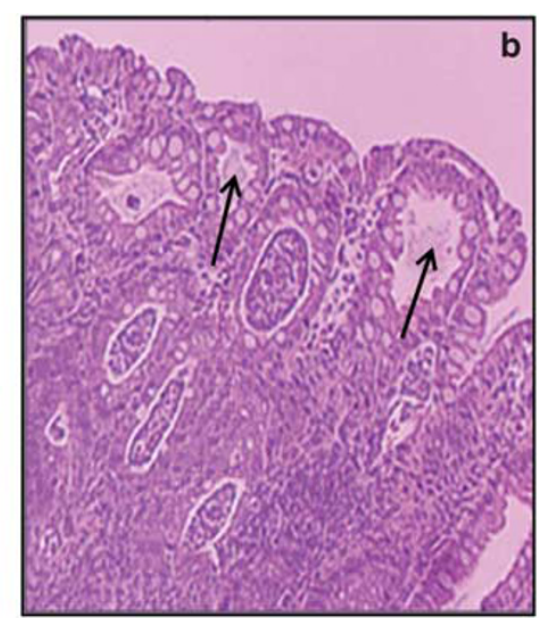

10 weeks old

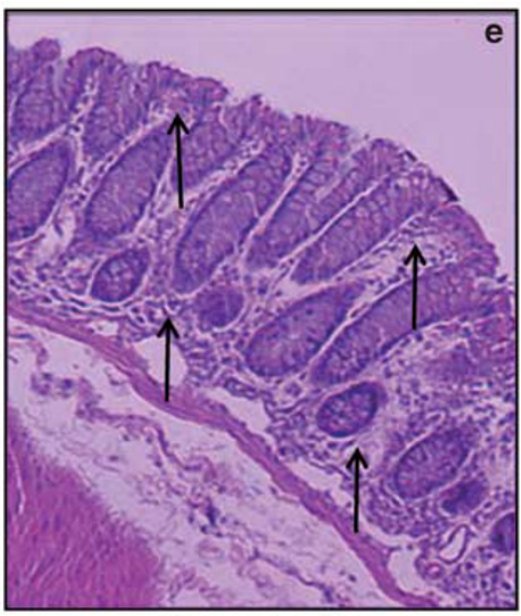

16 weeks old

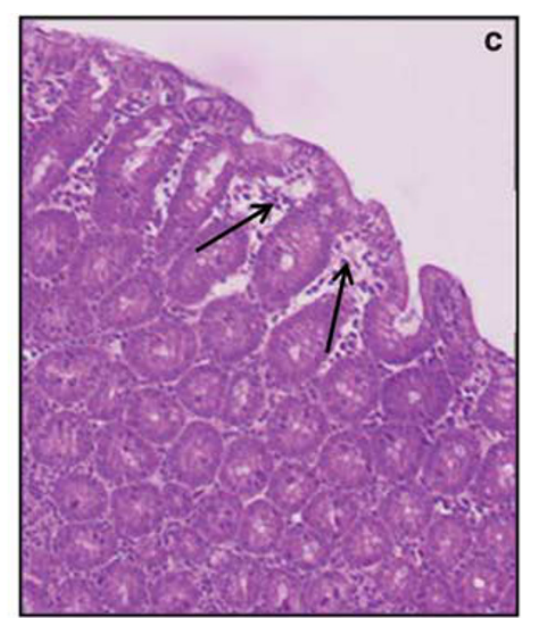

12 weeks old

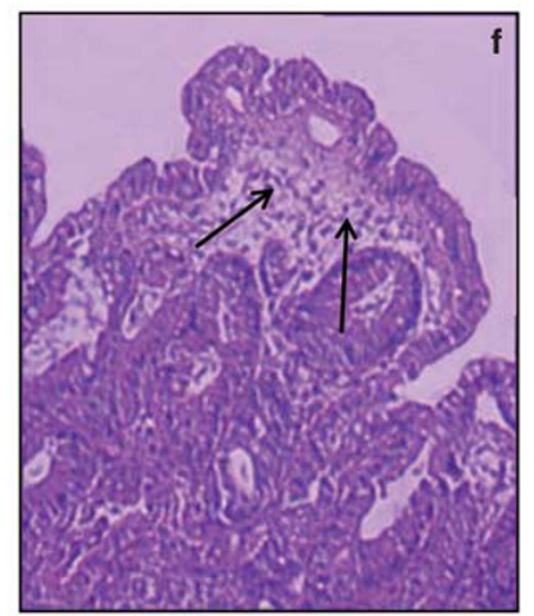

45 weeks old

Figure 8 Representative light microscopic photomicrographs of colonic mucosa in IL-10 $0^{-1-}$ and $\mathrm{G} \alpha-\mathrm{i} 2^{-1-}$ mice (H\&E staining, $\left.\times 100\right)$. (a) Wild-type control.

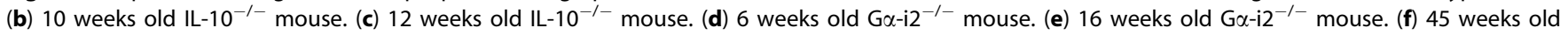
$\mathrm{G} \alpha-\mathrm{i} 2^{-1-}$ mouse. Areas of colonic mucosa with lamina propria edema (arrows) are covered by intact layer of the surface epithelial cells.

colonic tissue in early time points (at 15, $30 \mathrm{~min}$, and $1 \mathrm{~h}$ ) after IA administration before lesions developed and moreover before elevation of colonic EP. Furthermore, a recent study of Harris et al on chronic T-cell transfer model of IBD in mice confirmed our data and showed that Hypoxyprobe-1 staining was more prominent in the superficial area of the colonic mucosa. ${ }^{39}$ Harris et $a l^{40}$ study showed that mice exhibiting mild colonic inflammation had increased hypoxia, decreased systemic hematocrit, and reduced vascular density. Mice with severe colitis exhibited decreased hypoxia, higher levels of hematocrit and vascular density $v s$ mice with mild colonic inflammation. The hypoxia correlated inversely with hematocrit and vascular density. Therefore, the early stages of chronic colitis (mild inflammation) are associated with the hypoxia of epithelial cells that later results in erosions/ulcers development (severe colitis). In advanced colitis, increased angiogenesis likely triggered by initial hypoxia lead to increased vascularization and reduction of hypoxia. We also showed concomitant upregulation of hypoxia-related transcription factors HIF- $1 \alpha$ and Egr-1. Our data are in agreement with others on the models of UC induced by adoptive transfer of IL- $10^{-1-} \mathrm{CD} 4{ }^{+} \mathrm{T}$ cells into $\mathrm{RAG}^{-/-}$mice ${ }^{40}$ or TNBS in mice. ${ }^{41}$

Since UC is a chronic disease, characterized by frequent relapses, our findings of early increase in VP may be not only a reliable predictor of recurrence but also the basis for lesion development. In our study, we easily visualized extravasations of Evans blue as early as 1-2 days after DSS ingestion or $30 \mathrm{~min}$ to $12 \mathrm{~h}$ after IA enema as shown in Figure 1a, and blue color was strictly restricted to distal colon where DSS- $^{42}$ and IA-induced ${ }^{13,14}$ lesions develop. Edema of the mucosal lamina propria and infiltration by inflammatory cells are constant features of human UC, reflecting increased VP providing thus direct human relevance of our experimental studies. Moreover, recent studies from our group using confocal endomicroscopy demonstrated the presence 
Table 1 A summary of light microscopic evaluation of histologic changes in the colon of $\mathrm{G} \alpha-\mathrm{i} 2^{-I-}$ and IL-10 ${ }^{-I-}$ mice



Definitions of the categories:

Subepithelial edema: edema located under surface layer of the epithelial cells.

Lamina propria edema/inflammation: edema of the lamina propria and inflammatory cell infiltration.

Erosion: lesions localized to the mucosal layer.

Ulcer: lesions penetrating into submucosal layer.

of increased VP in patients with UC even among those in remission. ${ }^{43}$ Increased VP was associated with upregulation of vascular endothelial growth factor (VEGF), which is known as a potent VP factor. Increased VEGF levels during early stages of UC may provide a mechanistic clue to impaired endothelial barrier and increased VP. In our previous study, we demonstrated that neutralizing anti-VEGF antibody diminished colonic VP and improved clinical and morphologic signs of IA-UC, ${ }^{15}$ thus providing further support for the pathogenic role of increased VP in UC.

The precise mechanism(s) why and how endothelial permeability actually develops in UC remains unclear. There are several possibilities. It can be due to genetic predisposition, which impairs endothelial microvascular 'barrier.' The study of IL- $10^{-1-}$ mice showing increased lamina propria edema could support this contention. Second, it can be due to activation of VEGF gene and its product. VEGF is also known as VP factor. Other and our laboratories demonstrated activation of VEGF in UC, especially in its early phase, especially that neutralization of endogenous VEGF with specific anti-VEGF antibody abolishes increased endothelial microvascular permeability and prevents development of experimental UC. ${ }^{15}$ In addition to elevated VEGF, other non-VEGF mechanisms such as activation of TLR2 by oxidation stress can contribute to increased endothelial microvascular permeability in UC. ${ }^{44}$ TLR2 are present on some endothelial cells and TLR2 agonist increases endothelial monolayer permeability in vitro. ${ }^{45}$
In summary, the present study is the first demonstration of the role of colonic endothelial $v s$ epithelial barrier in the development of UC. These findings may contribute to a better understanding of the cellular/molecular mechanisms of UC pathogenesis, and provide new targets for the treatment and prevention of the relapses of this disease.

\section{ACKNOWLEDGEMENTS}

This work was supported by the Department of Veterans Affairs, Veterans Health Administration Merit Review Grants VAMR0710-580, and VAMR0810877 to Zs Sandor and S Szabo.

\section{DISCLOSURE/CONFLICT OF INTEREST}

The authors declare no conflict of interest.

1. Al-Sadi R, Boivin M, Ma T. Mechanism of cytokine modulation of epithelial tight junction barrier. Front Biosci 2009;4:2765-2778.

2. Mankertz J, Schulzke JD. Altered permeability in inflammatory bowel disease: pathophysiology and clinical implications. Curr Opin Gastroenterol 2007;23:379-383.

3. Farhadi A, Banan A, Fields J, et al. Intestinal barrier: an interface between health and disease. J Gastroenterol Hepatol 2003;18: 479-487.

4. Jump RL, Levine AD. Mechanisms of natural tolerance in the intestine: implications for inflammatory bowel disease. Inflamm Bowel Dis 2004;10:462-478.

5. Taylor CT, Colgan SP. Hypoxia and gastrointestinal disease. J Mol Med 2007;85:1295-1300.

6. Brahme F, Lindstrom C. A comparative radiographic and pathological study of intestinal vaso-architecture in Crohn's disease and in ulcerative colitis. Gut 1970;11:928-940.

7. Brahme F. Mesenteric angiography in regional enterocolitis. Radiology 1966;87:1037-1042. 
8. Chidlow Jr JH, Shukla D, Grisham MB, et al. Pathogenic angiogenesis in IBD and experimental colitis: new ideas and therapeutic avenues. Am J Physiol Gastrointest Liver Physiol 2007;293:5-18.

9. Hatoum OA, Miura H, Binion DG. The vascular contribution in the pathogenesis of inflammatory bowel disease. Am J Physiol Heart Circ Physiol 2003;285:1791-1796.

10. Foitzik T, Kruschewski M, Kroesen A, et al. Does microcirculation play a role in the pathogenesis of inflammatory bowel diseases? Answers from intravital microscopic studies in animal models. Int J Colorectal Dis 1999;14:29-34.

11. Berg DJ, Davidson N, Kühn R, et al. Enterocolitis and colon cancer in interleukin-10-deficient mice are associated with aberrant cytokine production and CD4(+) TH1-like responses. J Clin Invest 1996;98: 1010-1020.

12. Rudolph U, Finegold MJ, Rich SS, et al. Gi2 alpha protein deficiency: a model of inflammatory bowel disease. J Clin Immunol 1995;15: 101-105.

13. Sandor Z, Nagata $M$, Kusstatscher $S$, et al. New animal model of ulcerative colitis associated with depletion of glutathione and protein SH alkylation. Gastroenterology 1994;106:A766.

14. Satoh H, Sato F, Takami K, et al. New ulcerative colitis model induced by SH blockers in rats and the effects of antiinflammatory drugs on the colitis. Jpn J Pharmacol 1997;73:299-309.

15. Tolstanova G, Khomenko T, Deng X, et al. Neutralizing anti-vascular endothelial growth factor (VEGF) antibody reduces severity of experimental ulcerative colitis in rats: direct evidence for the pathogenic role of VEGF. J Pharmacol Exp Ther 2009;328:749-757.

16. Patterson CE, Rhoades RA, Garcia JG. Evans blue dye as a marker of albumin clearance in cultured endothelial monolayer and isolated lung. J Appl Physiol 1992;72:865-873.

17. McLaren WJ, Anikijenko P, Thomas SG, et al. In vivo detection of morphological and microvascular changes of the colon in association with colitis using fiberoptic confocal imaging (FOCl). Dig Dis Sci 2002;47:2424-2433.

18. Tarnawski A, Pai R, Deng $X$, et al. Aging gastropathy-novel mechanisms: hypoxia, up-regulation of multifunctional phosphatase PTEN, and proapoptotic factors. Gastroenterology 2007;133:1938-1947.

19. Shepherd AP. Local control of intestinal oxygenation and blood flow. Ann Rev Physiol 1982;44:13-27.

20. Linskens RK, Huijsdens XW, Savelkoul PH, et al. The bacterial flora in inflammatory bowel disease: current insights in pathogenesis and the influence of antibiotics and probiotics. Scand J Gastroenterol Supp 2001;234:29-40

21. Sun Z, Wang $X$, Andersson R. Role of intestinal permeability in monitoring mucosal barrier function. History, methodology, and significance of pathophysiology. Dig Surg 1998;15:386-397.

22. Szabo $S$, Trier JS, Brown A, et al. Early vascular injury and increased VP in gastric mucosal injury caused by ethanol in the rat. Gastroenterology 1985;88:228-236.

23. Szabo S, Vattay $P$, Scarbrough $E$, et al. Role of vascular factors, including angiogenesis, in the mechanisms of action of sucralfate. Am J Med 1991;91:158-160.

24. Prasad S, Mingrino R, Kaukinen $\mathrm{K}$, et al. Inflammatory processes have differential effects on claudins 2,3 and 4 in colonic epithelial cells. Lab Invest 2005;85:1139-1162.

25. Thornton M, Solomon MJ. Crohn's disease: in defense of a microvascular aetiology. Int J Colorectal Dis 2002;17:287-297.

26. Heidemann J, Domschke W, Kucharzik T, et al. Intestinal microvascular endothelium and innate immunity in inflammatory bowel disease: a second line of defense? Infect Immun 2006;74:5425-5432.
27. Danese S, Dejana E, Fiocchi C. Immune regulation by microvascular endothelial cells: directing innate and adaptive immunity, coagulation, and inflammation. J Immunol 2007:178:6017-6022.

28. Sydora BC, Tavernini MM, Doyle J, et al. A defect in epithelial barrier integrity is not required for a systemic response to bacterial antigens or intestinal injury in $\mathrm{T}$ cell receptor-alpha gene-deficient mice. Inflamm Bowel Dis 2006;12:750-757.

29. Oshima T, Laroux FS, Coe LL, et al. Interferon-gamma and interleukin10 reciprocally regulate endothelial junction integrity and barrier function. Microvasc Res 2001;61:130-143.

30. Su L, Shen L, Clayburgh DR, et al. Targeted epithelial tight junction dysfunction causes immune activation and contributes to development of experimental colitis. Gastroenterology 2009;136: 551-563.

31. Mori M, Salter JW, Vowinkel T, et al. Molecular determinants of the prothrombogenic phenotype assumed by inflamed colonic venules. Am J Physiol Gastrointest Liver Physiol 2005;288:920-926.

32. Horton WJ, Walker PB. Oxygen radicals, lipid peroxidation, and permeability changes after intestinal ischemia and reperfusion. J Appl Physiol 1993;74:1515-1520.

33. Rezaie A, Parker RD, Abdollahi M. Oxidative stress and pathogenesis of inflammatory bowel disease: an epiphenomenon or the cause? Dig Dis Sci 2007;52:2015-2021.

34. Wang $P, C h e n C L, L i ~ M$, et al. The role of myosin light chain kinase in intestinal epithelial barrier dysfunction due to hypoxia. Zhonghua Shao Shang Za Zhi 2009;25:57-60.

35. Synnestvedt K, Furuta GT, Comerford KM, et al. Ecto-5'-nucleotidase (CD73) regulation by hypoxia-inducible factor- 1 mediates permeability changes in intestinal epithelia. J Clin Invest 2002;110: 993-1002.

36. Hatoum OA, Binion DG, Gutterman DD. Paradox of simultaneous intestinal ischaemia and hyperaemia in inflammatory bowel disease. Eur J Clin Invest 2005;35:599-609.

37. Fries W, Pagiaro $E$, Canova $E$, et al. The effect of heparin on trinitrobenzene sulphonic acid-induced colitis in the rat. Aliment Pharmacol Ther 1998;12:229-236.

38. Kruschewski $M$, Anderson $T$, Loddenkemper $C$, et al. Endothelin-1 receptor antagonist (LU-135252) improves the microcirculation and course of TNBS colitis in rats. Dig Dis Sci 2006;51:1461-1470.

39. Colgan SP, Taylor CT. Hypoxia: an alarm signal during intestinal inflammation. Nat Rev Gastroenterol Hepatol 2010;7:281-287.

40. Harris NR, Carter PR, Yadav AS, et al. Relationship between inflammation and tissue hypoxia in a mouse model of chronic colitis. Inflamm Bowel Dis 2011;17:742-746.

41. Karhausen J, Furuta GT, Tomaszewski JE, et al. Epithelial hypoxiainducible factor- 1 is protective in murine experimental colitis. J Clin Invest 2004;114:1098-1106.

42. Gaudio E, Taddei G, Vetuschi A, et al. Dextran sulfate sodium (DSS) colitis in rats: clinical, structural, and ultrastructural aspects. Dig Dis Sci 1999;44:1458-1475.

43. Tarnawski A, Coron E, Mosnier JF, et al. In-vivo detection by confocal endomicroscopy of two distinct structural abnormalities in angioarchitecture and increased VP in colonic mucosa of patients with IBD in remission: mechanistic implications. Gastroenterology 2009;136:A112.

44. West XZ, Malinin NL, Merkulova AA, et al. Oxidative stress induces angiogenesis by activating TLR2 with novel endogenous ligands. Nature 2010;467:972-976.

45. Shin HS, Xu F, Bagchi A, et al. Bacterial lipoprotein TLR2 agonists broadly modulate endothelial function and coagulation pathways in vitro and in vivo. J Immunol 2011;186:1119-1130. 